\title{
Diurnal frequency internal waves in the southern part of the California Current ecosystem as a nutrient source
}

\section{Ondas internas diurnas en la parte sur del ecosistema de la corriente de California como fuente de nutrientes}

\author{
Lydia Betty Ladah ${ }^{1 *}$, James J Leichter ${ }^{2}$, Anatoliy Filonov ${ }^{3}$, Iryna Tereshchenko ${ }^{3}$ \\ ${ }^{1}$ Department of Biological Oceanography, Centro de Investigación Científica y de Educación Superior de \\ Ensenada (CICESE), Carretera Ensenada-Tijuana, no. 3918, CP 22860, Zona Playitas, Ensenada, Baja \\ California, Mexico. \\ 2 Integrative Oceanography Division, Scripps Institution of Oceanography m/c 0227, 9500 Gilman Drive, La \\ Jolla 92093-0227, California, USA. \\ 3 Physics Department, Universidad de Guadalajara, Blvd. Marcelino Garcia Barragan \# 1421, Guadalajara, \\ Mexico. \\ * Corresponding author. E-mail: 1ladah@cicese.mx
}

\begin{abstract}
Although the semidiurnal internal tide dominates internal motions at all sites studied to date off Mexico, a diurnal signal was detected on the shelf of the California Current System off Baja California Sur, even though the barotropic tide is exponentially stronger in the semidiurnal frequency along this coastline. In this study, diurnal thermocline shoaling events were measured in kelp beds in late summer and fall of 2004, very near their southern limit in the Northern Hemisphere. Sub-thermocline water bathed the base of the kelp beds at a diurnal frequency. Nitrate concentrations up to $1.4 \mu \mathrm{mol} \cdot \mathrm{L}^{-1}$ were found in this sub-thermocline water of almost $20^{\circ} \mathrm{C}$ entering the $\mathrm{kelp}$ beds, which is a much greater concentration of nitrate at this temperature than is typical for the California Current. Nitrate-rich pulses lasted from 35 min to $19 \mathrm{~h}$ and reduced bottom temperatures up to $5^{\circ} \mathrm{C}$ in $1 \mathrm{~h}$. The periodicity of temperature variability at the moorings showed significant coherence with the diurnal variability in zonal winds ( $>95 \%$ confidence interval) and was not coherent with the semidiurnal tide. We propose that windforced, diurnal internal motions may provide an important nutrient source for primary producers during reduced upwelling periods and merit further study as a fertilization source in the southern part of the California Current System.
\end{abstract}

Key words: Baja California, Mexico, internal waves, nutrients, Gulf of Ulloa.

RESUMEN. A pesar de que la marea semidiurna predomina en las ondas internas de casi todos los sitios estudiados hasta la fecha en México, se detectó una marea interna diurna sobre la plataforma continental del Sistema de la Corriente de California frente a las costas de Baja California Sur, aunque la marea barotrópica es exponencialmente más fuerte en la frecuencia semidiurna. En este estudio, se midió la subida diurna de la termoclina en los mantos de kelp, localizados cerca del límite sureño de su distribución en el hemisferio norte, durante el verano y el otoño de 2004. Agua profunda cubrió la base de los mantos de kelp una vez al día donde se encontraron concentraciones de nitrato de hasta $1.4 \mu \mathrm{mol} \cdot \mathrm{L}^{-1}$ en agua de casi $20^{\circ} \mathrm{C}$, lo cual representa una concentración elevada de nitrato a esa temperatura para la corriente de California. Los pulsos de agua rica en nitrato duraron de $35 \mathrm{~min}$ a $19 \mathrm{~h}$ en los mantos y redujeron la temperatura del fondo hasta $5{ }^{\circ} \mathrm{C}$ en $1 \mathrm{~h}$. La periodicidad de la variabilidad de la temperatura mostró una coherencia significativa con la variabilidad diurna de los vientos zonales ( $>95 \%$ intervalo de confianza) y no fue coherente con la marea semidiurna. Proponemos que las ondas internas diurnas, forzadas por el viento, podrían proveer una fuente importante de nutrientes a los productores primarios durante periodos en los cuales la actividad de surgencias es reducida y requieren de más estudio para evaluar su capacidad de actuar como una fuente de fertilización en la parte sur del Sistema de la Corriente de California.

Palabras clave: Baja California, México, ondas internas, nutrientes, golfo de Ulloa

\section{INTRODUCTION}

The California Current is a highly productive eastern boundary current, spanning temperate and subtropical latitudes along the west coast of the United States and the Baja California Peninsula in Mexico. The latitudinal range of this current is dominated by a semidiurnal mixed barotropic tide and bridges the critical latitude of $30^{\circ} \mathrm{N}$ for diurnal internal waves, north of which, theoretically, these waves cannot exist as freely propagating internal waves. The inner shelf of most of the California Current System is dominated

\section{INTRODUCCIÓN}

La corriente de California es una corriente de frontera oriental de alta productividad, y abarca latitudes templadas y subtropicales a lo largo de la costa occidental de los Estados Unidos y de la península de Baja California, México. A lo largo del rango latitudinal de esta corriente, domina la marea barotrópica mixta semidiurna. La corriente de California cruza la latitud crítica de $30^{\circ} \mathrm{N}$ para ondas internas diurnas, al norte de la cual, teóricamente, estas ondas no pueden existir como ondas internas de propagación libre. Sobre la plataforma continental del 
by conspicuous kelp forests, with their southern range limit extending to Baja California Sur, Mexico. Although for most of the California Current System upwelling provides sufficient nutrients for primary producers year round, other mechanisms of sub-thermocline water transport, such as internal waves, have recently been shown to be important in the Mexican part of the California Current System (northern Baja California) (Ladah et al. 2012) and may be particularly important for kelp forests at their southern limit of distribution in the Northern Hemisphere off Baja California Sur.

The transport of sub-thermocline nutrients by internal waves may be an alternative mechanism for the provision of nutrients in seasons or in regions of reduced upwelling. Internal waves can force vertical and horizontal transport of many scalars, such as particles, larvae, sub-thermocline water, and nutrients for primary producers (Franks 1997, Ladah et al. 2012, Salas-Monreal et al. 2012), and are prevalent features where flows encounter sharp bathymetry in stratified waters (Holloway 1987, Lamb 1997). As internal waves propagate shoreward and touch the bottom, they can increase in amplitude and become nonlinear internal waves (NLIWs), causing rapid physical and chemical changes in shallow water (Franks 1992, Helfrich and Pineda 2003, Helfrich and Melville 2006) and strong diapycnal mixing, with dissipation rates reaching $0.2 \mathrm{~W} \cdot \mathrm{m}^{-2}$ over a short distance (Sandstrom and Elliot 1984, 2011).

The supply of inorganic nutrients has been directly linked with NLIWs (Sandstrom and Elliot 1984; Leichter et al. 1996, 2003; Sharples et al. 2001, 2009), which can account for a diapycnal flux of nitrate greater than $3 \mathrm{mmol} \cdot \mathrm{m}^{-2} \cdot \mathrm{d}^{-1}$ (Sharples et al. 2007, Schafstall et al. 2010), up to $25 \%$ of the annual nitrate contribution on the shelf in the California Current off Santa Barbara (McPhee-Shaw et al. 2007), and increased primary productivity on the inner shelf of the California Current off Southern California (Lucas et al. 2011). The only site to date where internal waves have been explored in the Mexican part of the California Current System is Todos Santos Bay in Northern Baja California (Filonov et al. 2014), where macroalgal tissue nitrogen doubled in response to semidiurnal internal wave nutrient pulses within a few hours (Pérez-Mayorga et al. 2011, Ladah et al. 2012). However, there are no in situ measurements of internal wave activity in any other site of the California Current system off Mexico, particularly in the more subtropical zone where mesoscale wind-induced upwelling becomes infrequent and less intense (Kurzyn et al. 2012) and nutrient provision by internal waves may be even more important for primary producers.

In San Juanico, Baja California Sur, Apel and Gonzalez (1983) documented internal waves using satellite imagery. This site is very near the southern limit of all kelp species in the Northern Hemisphere. Here, perennial individuals of the southern sea palm, Eisenia arborea (now known as Ecklonia arborea), thrive even when sea surface temperature reaches $26^{\circ} \mathrm{C}$, which is presumably a nutrient-poor condition (Ladah 2003). Although the ability of kelps to survive high
Sistema de la Corriente de California predominan los bosques de kelp, cuyo límite sureño se extiende hasta Baja California Sur, México. A pesar de que las surgencias del Sistema de la Corriente de California proveen nutrientes para los productores primarios a lo largo del año, recientemente se ha demostrado que otros mecanismos de transporte de agua profunda hacia zonas someras, como las ondas internas, son importantes en la parte mexicana de la corriente de California (norte de Baja California) (Ladah et al. 2012) y podrían ser de importancia particular para los bosques de kelp en el límite sur de su distribución en el hemisferio norte, frente a las costas de Baja California Sur.

El transporte de nutrientes desde aguas profundas hacia zonas someras llevado a cabo por ondas internas puede ser un mecanismo alterno para proveer nutrientes durante temporadas o en regiones que presentan una disminución en la actividad de surgencias. Las ondas internas pueden forzar el transporte vertical y horizontal de partículas, larvas, agua profunda y nutrientes para los productores primarios (Franks 1997, Ladah et al. 2012, Salas-Monreal et al. 2012), y son prevalentes en sitios con aguas estratificadas y batimetrías abruptas (Holloway 1987, Lamb 1997). Cuando las ondas internas se propagan hacia la costa y tocan el fondo, pueden incrementar su amplitud y volverse ondas internas no lineales (OINL), provocando cambios físicos y químicos rápidos en aguas someras (Franks 1992, Helfrich y Pineda 2003, Helfrich y Melville 2006) y una fuerte mezcla diapicnal, con tasas de disipación que alcanzan hasta $0.2 \mathrm{~W} \cdot \mathrm{m}^{-2}$ en distancias cortas (Sandstrom y Elliot 1984, 2011).

El suministro de nutrientes inorgánicos se ha ligado de manera directa a la actividad de OINL (Sandstrom y Elliot 1984; Leichter et al. 1996, 2003; Sharples et al. 2001, 2009), lo que puede explicar un flujo diapicnal de nitrato mayor que $3 \mathrm{mmol} \cdot \mathrm{m}^{-2} \cdot \mathrm{d}^{-1}$ (Sharples et al. 2007, Schafstall et al. 2010), hasta el $25 \%$ de la contribución anual de nitrato para la plataforma del Sistema de la Corriente de California frente a las costas de Santa Barbara (McPhee-Shaw et al. 2007), y puede incrementar la productividad primaria sobre la plataforma continental frente a las costas del sur de California (Lucas et al. 2011). Hasta la fecha, el único sitio en el cual las ondas internas han sido exploradas en la parte mexicana del Sistema de la Corriente de California es la bahía de Todos Santos, ubicada en la parte norte de Baja California (Filonov et al. 2014), donde la concentración de nitrógeno en los tejidos de macroalgas se duplicó en pocas horas en respuesta a los pulsos de nutrientes de ondas internas semidiurnas (Pérez-Mayorga et al. 2011, Ladah et al. 2012). Sin embargo, no existen mediciones in situ de la actividad de las ondas internas en ningún otro sitio del Sistema de la Corriente de California en México, particularmente en la zona más subtropical donde las surgencias de mesoescala forzadas por el viento se vuelven menos frecuentes e intensas (Kurzyn et al. 2012) y la provisión de nutrientes por ondas internas podría ser aún más importante para los productores primarios.

En San Juanico, Baja Californa Sur, Apel y Gonzalez (1983) documentaron las ondas internas utilizando imágenes 
temperatures depends on multiple factors, survival is closely tied to tissue nitrogen concentrations (Gerard 1982, 1997) and ambient nitrogen availability (Gerard 1984; Zimmerman and Kremer 1984, 1986). Ecklonia arborea shows a significant reduction in tissue nitrogen to below $1 \%$ dry weight in about $20 \mathrm{~d}$ when cultured in nutrient-poor outdoor tanks or when exposed to nutrient-poor warm temperatures (Matson and Edwards 2007, Sánchez-Barredo et al. 2011). Therefore, the detection of $E$. arborea thriving in warm waters, with tissue nitrogen as high as $2.5 \%$, in summer off San Juanico suggests that these kelps are exposed to a nutrient source even during warm sea surface temperatures.

In this study, we show that diurnal internal waves dominate at our study site and discuss the forcing mechanisms and potential importance for the ecology of the inner shelf of the California Current System off Baja California Sur, where upwelling is reduced in late summer and fall compared with other sites along the California Current. In this contribution, we investigate the potential factors controlling variability in high-frequency temperature records collected in the summer and fall seasons of the weak 2004-2005 El Niño event. The motivation for this study arises from observations of healthy kelp beds with high tissue nitrogen (above $2.5 \%$ dry weight) during warm summer conditions. We propose that nutrient pulses associated with diurnal internal waves may be important to the summer survival of kelp beds in this region and speculate on their forcing. This study highlights the fact that the southern part of the California Current System is a biogeographic transition zone and suggests that mechanisms that may not be particularly important in one region may be highly relevant in another, considering the great latitudinal span of the California Current.

\section{MATERIALS AND METHOdS}

\section{Study site}

San Juanico $\left(26.30285^{\circ} \mathrm{N}, 112.62340^{\circ} \mathrm{W}\right)$ is located in one of the largest and shallowest areas along the California Current, in the Gulf of Ulloa, at the southern end of the California Current System, on the Pacific coast of Baja California Sur. This area is very near the southern limit of the kelp E. arborea in the Northern Hemisphere (Fig. 1). This site is characterized by strong local winds, a very wide, gently sloping shelf, and dominant semidiurnal tides ranging from -0.26 to $2.24 \mathrm{~m}$.

\section{Water column temperature and nutrients}

Temperature loggers sampling every $5 \min \left(0.2^{\circ} \mathrm{C}\right.$ accuracy, approximately $5 \mathrm{~min}$ response time; Onset Computer Corp., MA, USA) were installed on vertical mooring lines deployed at the 8-, 15-, and 20-m bathymetric contours (see Fig. 1b). The first 2 mooring lines ( 8 and $15 \mathrm{~m}$ ) were adjacent to perennial E. arborea kelp beds, and the 20-m mooring line de satélite. Este sitio se encuentra cerca del límite sur de todas las especies de kelp en el hemisferio norte. Aquí, los individuos perennes de la palmera de mar sureña, Eisenia arborea (ahora conocida como Ecklonia arborea), prosperan aun cuando la temperatura superficial del mar alcanza los $26^{\circ} \mathrm{C}$, que supuestamente es una condición donde la concentración de nutrientes es pobre (Ladah 2003). A pesar de que la capacidad del kelp para sobrevivir temperaturas altas depende de varios factores, la supervivencia está altamente ligada a la concentración de nitrógeno en los tejidos (Gerard 1982, 1997) y a la disponibilidad de nitrógeno en el ambiente (Gerard 1984; Zimmerman y Kremer 1984, 1986). Ecklonia arborea muestra una reducción significativa de nitrógeno en sus tejidos, hasta menos del $1 \%$ de peso seco en $20 \mathrm{~d}$, cuando se cultiva en estanques al aire libre que tienen una concentración de nutrientes baja o cuando se expone a aguas cálidas y escasas de nutrientes (Matson y Edwards 2007, Sánchez-Barredo et al. 2011). Por lo tanto, la detección de poblaciones de E. arborea en aguas cálidas, con un porcentaje de nitrógeno en los tejidos de hasta $2.5 \%$, durante el verano en San Juanico sugiere que estos kelps están expuestos a una fuente de nutrientes aun cuando se presentan temperaturas cálidas.

En este estudio, demostramos que las ondas internas diurnas dominan en el sitio de estudio y discutimos sus mecanismos de forzamiento e importancia potencial para la ecología del Sistema de la Corriente de California sobre la plataforma continental frente a las costas de Baja California Sur, donde las surgencias se reducen a finales de verano y otoño en comparación con otros sitios a lo largo de la corriente de California. En esta contribución, investigamos los factores potenciales que controlan la variabilidad de alta frecuencia en las series de tiempo de temperatura recolectados en las temporadas de verano y otoño durante el evento débil de El Niño de 2004-2005. La motivación para este estudio surge de las observaciones de mantos de kelp sanos con altas concentraciones de nitrógeno en su tejido (arriba de 2.5\%, peso seco) durante las condiciones cálidas de verano. Proponemos que los pulsos de nitrógeno asociados a la actividad de ondas internas diurnas pueden ser importantes para la supervivencia de los mantos de kelp durante el verano en esta región y especulamos acerca de sus mecanismos de forzamiento. Este estudio destaca el hecho de que la parte sur del Sistema de la Corriente de California es una zona de transición biogeográfica y sugiere que los mecanismos que tal vez no son de mucha importancia en unas regiones pueden ser de alta importancia en otras regiones, considerando que la corriente de California abarca una gran extensión latitudinal.

\section{MATERIALES Y MÉTODOS}

\section{Sitio de estudio}

San Juanico $\left(26.30285^{\circ} \mathrm{N}, 112.62340^{\circ} \mathrm{W}\right)$ se localiza en el golfo de Ulloa y se encuentra en una de las áreas más largas y someras de la parte sur de la corriente de California, sobre la costa del Pacífico de Baja California Sur. Esta área se ubica 
was $3 \mathrm{~km}$ offshore. Loggers were installed every meter on the shallowest mooring and every $3 \mathrm{~m}$ on the deeper moorings.

Water column hydrography was measured with vertical casts using a SeaBird Electronics 19 ConductivityTemperature-Depth (CTD) profiler on 14-16 August and 2-6 September 2004 along an offshore transect encompassing the thermistor lines (see Fig. 1). Water samples were collected using 1.2-L Niskin bottles at the surface, mid-water, and $1 \mathrm{~m}$ above the CTD at its maximum depth; they were then filtered (GFF, $0.7 \mu \mathrm{m}$ ), frozen, and analyzed in triplicate for dissolved inorganic nitrogen using an autoanalyzer with minimal detectable limits at $0.2 \mu \mathrm{mol} \cdot \mathrm{L}^{-1}$. Nitrate and nitrite were graphed against temperature (Zentara and Kamykowski 1977) to examine the temperature-nutrient relationship for this area in summer (Ladah 2003).

\section{Wind and tide data}

Hourly wind data were available from a meteorological station maintained by the Comisión Federal de Electricidad (Federal Electricity Commission, Mexico), 14 km SE from the study site. Hourly tidal information was available from San Carlos (160 km south; $24^{\circ} 47^{\prime} \mathrm{N}, 112^{\circ} 07^{\prime} \mathrm{W}$ ).

\section{Analysis of temperature and wind data}

The vertical displacement of isotherms was calculated from the temperature time series by using the equation (Filonov and Konyaev 2003)

$$
\xi(t)=d T(t) / \overline{d T / d z}
$$

where $\xi(t)$ is the vertical displacement at a given depth $z$ at time $t$ and $\overline{d T / d z}$ is the average vertical temperature gradient.

Using 27 CTD casts from 14-16 August, the distribution of the average buoyancy frequency (Brunt-Väisälä frequency) had a minimum period of $2.7 \mathrm{~min}$, at $13 \mathrm{~m}$ depth, with the average period for the 5-25 m depths of 6 min (Fig. 1c). Because thermistors sampled every 5 min (a Nyquist period of $10 \mathrm{~min}$ ) it was not possible to resolve events on the order of minutes. The temperature time series were therefore smoothed with a 1-h sliding cosine filter.

Hourly wind data were analyzed for variability of the zonal (East-West) and meridional (North-South) wind components. For the wind time-series we applied rotational spectral analysis and cross spectral methods (Gonella 1972, Konyaev 1990, Emery and Thomson 1997). Squared coherence was calculated between temperature measured at all depths on mooring line A (within the shallow kelp bed) and (1) the dominant East-West component of the wind (24 h) or (2) the $\mathrm{O}_{1}$ $(25.82 \mathrm{~h})$ and $\mathrm{M}_{2}(12.42 \mathrm{~h})$ tidal sea level. We calculated the spectra of the temperature time series from 6 depths at thermistor mooring lines $\mathrm{A}, \mathrm{B}$, and $\mathrm{C}$ and for hourly sea level fluctuations of the same length ( $43 \mathrm{~d} ; 1,022$ hourly values). cerca del límite sureño de la distribución del kelp E. arborea en el hemisferio norte (Fig. 1). Este sitio se caracteriza por vientos locales fuertes, por una plataforma ancha y con pendiente suave, y por mareas semidiurnas dominantes con un rango de -0.26 a $2.24 \mathrm{~m}$.

\section{Temperatura de la columna de agua y nutrientes}

Sensores de temperatura programados para medir la temperatura cada $5 \mathrm{~min}$ (exactitud de $0.2^{\circ} \mathrm{C}$, tiempo de respuesta de aproximadamente $5 \mathrm{~min}$; Onset Computer Corp., MA, EUA) fueron colocados sobre líneas que se instalaron verticalmente en el mar en los contornos batimétricos de 8, 15 y $20 \mathrm{~m}$ (ver Fig. 1b). Las primeras 2 líneas (8 y $15 \mathrm{~m}$ ) se encontraron adyacentes a mantos perennes de $E$. arbórea, y la tercera línea $(20 \mathrm{~m})$ se instaló a $3 \mathrm{~km}$ de la costa. Los sensores de temperatura se colocaron cada metro sobre la línea más somera y cada $3 \mathrm{~m}$ sobre las líneas más profundas.

Se midió la hidrografía de la columna de agua mediante lances verticales con un perfilador SeaBird Electronics 19 Conductivity-Temperature-Depth (CTD) del 14 al 16 de agosto y del 2 al 6 de septiembre de 2004, a lo largo de un transecto mar adentro que abarcó las 3 líneas de termistores (ver Fig. 1). Se recolectaron muestras de agua utilizando botellas Niskin de $1.2 \mathrm{~L}$ en la superficie, a media agua y a $1 \mathrm{~m}$ arriba de la profundidad máxima del CTD; posteriormente, las muestras de agua fueron filtradas (GFF, $0.7 \mu \mathrm{m}$ ), congeladas y analizadas por triplicado para evaluar la cantidad de nitrógeno inorgánico disuelto utilizando un autoanalizador con un límite inferior de detección de $0.2 \mu \mathrm{mol} \cdot \mathrm{L}^{-1}$. Se graficaron el nitrato y el nitrito contra la temperatura (Zentara y Kamykowski 1977) para evaluar la relación entre la temperatura y la concentración de nutrientes para el área de estudio durante el verano (Ladah 2003).

\section{Datos de viento y marea}

Se obtuvieron los datos de viento para cada hora de la estación meteorológica de la Comisión Federal de Electricidad (México), ubicada $14 \mathrm{~km}$ al sureste del sitio de estudio. Los datos de marea para cada hora se obtuvieron de San Carlos $\left(160 \mathrm{~km}\right.$ al sur; $\left.24^{\circ} 47^{\prime} \mathrm{N}, 112^{\circ} 07^{\prime} \mathrm{W}\right)$.

\section{Análisis de datos de temperatura y viento}

El desplazamiento vertical de las isotermas se calculó utilizando la serie de tiempo de temperatura con la ecuación (Filonov y Konyaev 2003)

$$
\xi(t)=d T(t) / \overline{d T / d z}
$$

donde $\xi(t)$ es el desplazamiento vertical a una profundidad $z$ en el tiempo $t$ y $\overline{d T / d z}$ es el gradiente de temperatura vertical promedio. 


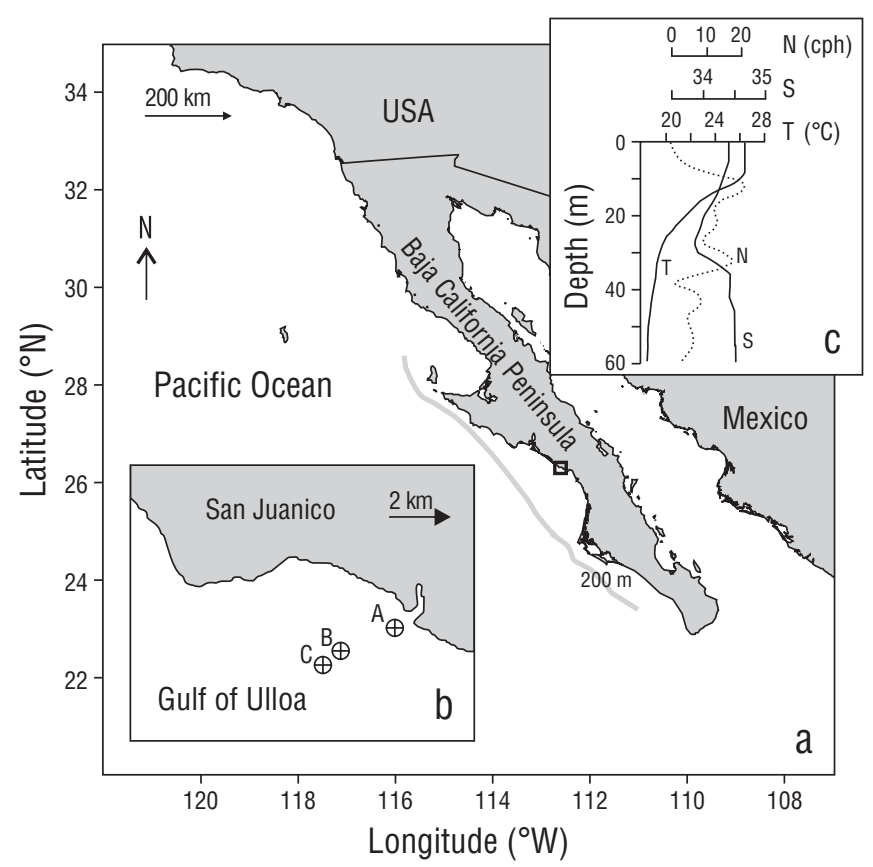

Figure 1. (a) Regional map of Baja California with a light grey bar showing the position of the 200-m isobath. (b) Inset showing the study area at San Juanico and the location of the instrument moorings. Sites A and B indicate thermistor mooring lines and kelp beds at 8 and $15 \mathrm{~m}$ depth; Site $\mathrm{C}$ corresponds to the offshore thermistor line moored at $20 \mathrm{~m}$ depth. (c) Inset showing vertical distributions of temperature (T), salinity (S), and buoyancy frequency $(\mathrm{N})$ near mooring line $\mathrm{C}$.

Figura 1. (a) Mapa regional de Baja California con una barra color gris que indica la posición de la isóbata de 200 m. (b) Recuadro que muestra el área de estudio de San Juanico y la ubicación de los instrumentos instalados. Los sitios A y B indican las líneas de termistores en los mantos de kelp a 8 y 15 m de profundidad; el sitio $\mathrm{C}$ corresponde a la línea de termistores mar adentro instalada a $20 \mathrm{~m}$ de profundidad. (c) Recuadro que muestra las distribuciones verticales de temperatura $(\mathrm{T})$, salinidad $(\mathrm{S})$ y la frecuencia de flotabilidad $(\mathrm{N})$ cerca de línea $\mathrm{C}$.

\section{RESULTS}

The temperature time series shows daily fluctuations reaching $5{ }^{\circ} \mathrm{C}$ (giving a calculated vertical displacement using equation [1] of approximately $18 \mathrm{~m}$ ) in mid-September (Fig. 2). The maximum water temperature was recorded on 10 September, when it exceeded $28^{\circ} \mathrm{C}$ at the surface (Fig. 2 a, b). Cooler water $\left(<20^{\circ} \mathrm{C}\right)$ episodically intruded into the kelp beds and reduced bottom temperature up to $5^{\circ} \mathrm{C}$ in an hour during the study period (Fig. 2c-e).

Water sampled at $19.7^{\circ} \mathrm{C}$ contained measurable nitrate plus nitrite $(1.96 \mu \mathrm{M})$, with an average of $5.45 \pm 4.05 \mu \mathrm{mol} \cdot \mathrm{L}^{-1}$ between 19.2 and $19.7^{\circ} \mathrm{C}$. Nitrate concentrations up to $1.4 \mu \mathrm{mol} \cdot \mathrm{L}^{-1}$, in waters of almost $20^{\circ} \mathrm{C}$, were found in this sub-thermocline water entering the kelp beds, which is a much greater concentration of nitrate at this temperature than is usually observed for the California Current.
Utilizando 27 lances de CTD tomados del 14 al 16 de agosto, la distribución de la frecuencia de flotabilidad promedio (frecuencia de Brunt-Väisälä) mostró un periodo mínimo de $2.7 \mathrm{~min}$ a $13 \mathrm{~m}$ de profundidad, con un periodo promedio de 6 min para las profundidades de 5 a $25 \mathrm{~m}$ (Fig. 1c). Dado que los termistores se programaron para medir la temperatura cada 5 min (periodo Nyquist de $10 \mathrm{~min}$ ), no fue posible resolver eventos a la escala de minutos. La serie de tiempo de temperatura se suavizó con un filtro de coseno móvil con una ventana de $1 \mathrm{~h}$.

Se analizaron los datos de viento de cada hora para evaluar la variabilidad del componente zonal (este-oeste) y del componente meridional (norte-sur). Para la serie de tiempo de viento, se aplicaron un análisis espectral rotacional y métodos espectrales cruzados (Gonella 1972, Konyaev 1990, Emery y Thomson 1997). El cuadrado de la coherencia se calculó entre la temperatura medida en cada profundidad a lo largo de la línea A (adentro del manto de kelp somero) y (1) el componente dominante este-oeste del viento (24 h) o (2) el nivel de la marea $\mathrm{O}_{1}(25.82 \mathrm{~h})$ y $\mathrm{M}_{2}(12.42 \mathrm{~h})$. Calculamos el espectro de la serie de tiempo de temperatura en 6 profundidades para las líneas A, B y C y también para las fluctuaciones del nivel del mar de la misma longitud (43 d; 1,022 valores cada hora).

\section{Resultados}

La serie de tiempo de temperatura mostró fluctuaciones diarias que alcanzaron $5^{\circ} \mathrm{C}$ (dado un desplazamiento vertical calculado utilizando la ecuación [1] de aproximadamente $18 \mathrm{~m}$ ) a mediados de septiembre (Fig. 2). La temperatura máxima del agua de mar se registró el 10 de septiembre, cuando alcanzó más de $28^{\circ} \mathrm{C}$ en la superficie (Fig. 2a, b). Agua más fría $\left(<20^{\circ} \mathrm{C}\right)$ se introdujo de manera episódica a los mantos de kelp y redujo la temperatura hasta $5^{\circ} \mathrm{C}$ en $1 \mathrm{~h}$ durante el periodo de estudio (Fig. 2c-e).

$\mathrm{El}$ agua recolectada a $19.7^{\circ} \mathrm{C}$ contuvo una concentración medible de nitrato más nitrito $(1.96 \mu \mathrm{M})$, con un promedio de $5.45 \pm 4.05 \mu \mathrm{mol} \cdot \mathrm{L}^{-1}$ entre 19.2 y $19.7^{\circ} \mathrm{C}$. Se encontraron concentraciones de nitrato de hasta $1.4 \mu \mathrm{mol} \cdot \mathrm{L}^{-1}$ en agua de casi $20^{\circ} \mathrm{C}$ que entraba a los mantos de kelp, lo cual representa una mayor concentración de nitrato a esta temperatura de lo que se observa usualmente en la corriente de California.

Después de un mes, la temperatura en el fondo presentó un promedio de $23.6^{\circ} \mathrm{C}$ en la base del manto de $8 \mathrm{~m}$ y $22.6{ }^{\circ} \mathrm{C}$ en la base del manto de $15 \mathrm{~m}$; las hojas de E. arborea contenían una concentración relativamente alta de nitrógeno $(2.37 \% \pm$ 0.19 a $8 \mathrm{~m}, 2.40 \% \pm 0.30$ a $15 \mathrm{~m}$, máximo de $2.85 \%$ a $8 \mathrm{~m}$ ). Dado que el porcentaje de nitrógeno en el tejido de kelp que presenta limitación por nutrientes es de alrededor del $1 \%$, el valor medido sugiere que el tejido de kelp no presentó limitación por nutrientes. El porcentaje de nitrógeno, carbono $(32.39 \% \pm 1.49,31.67 \% \pm 1.68)$ o hidrógeno $(3.97 \% \pm 0.28$, $4.16 \% \pm 0.27)$ no varió de manera significativa entre 8 y $15 \mathrm{~m}$ de profundidad $(P>0.15$, prueba $t$ de Student, $n=10)$. 

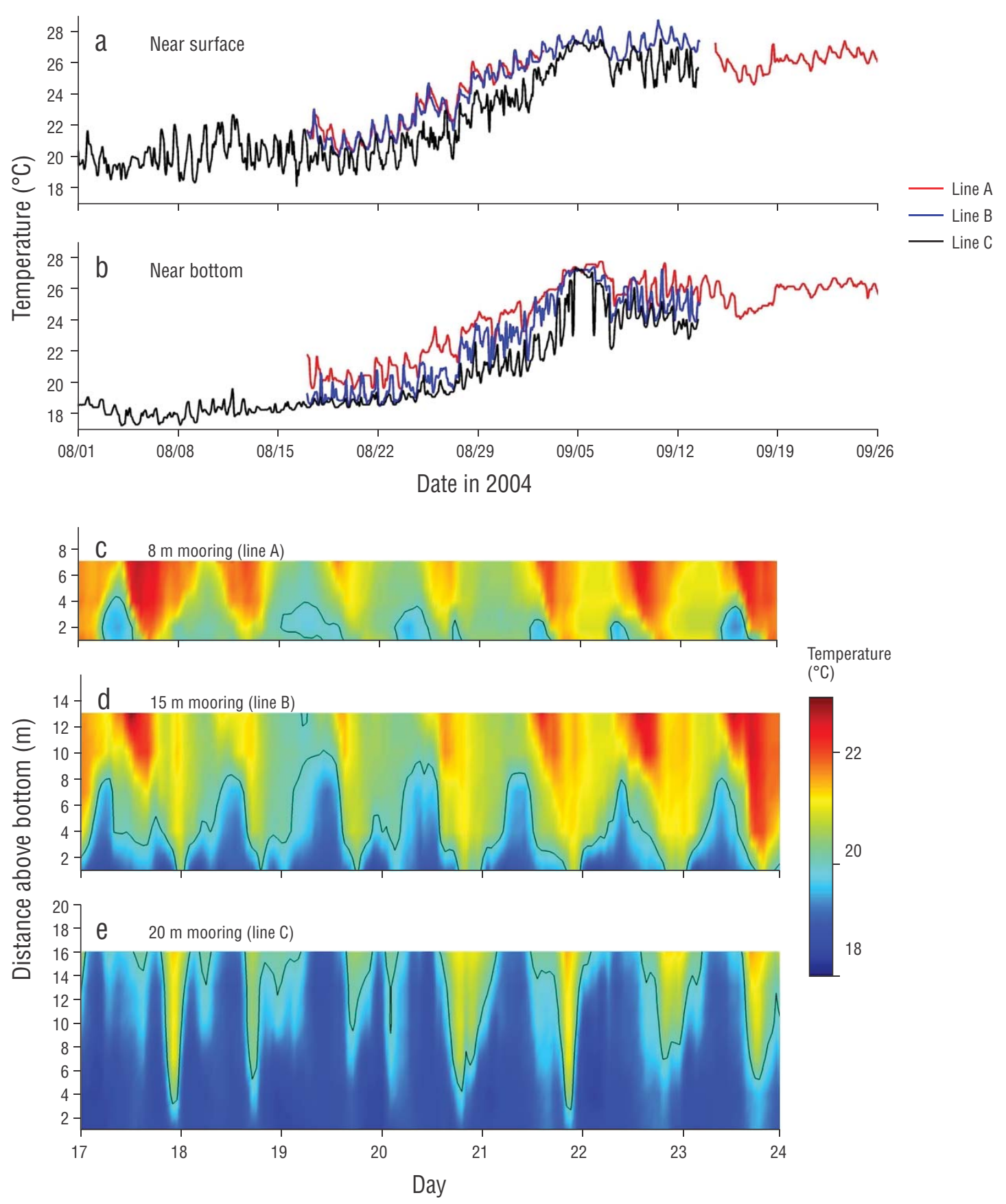

Figure 2. (a) Near-surface temperatures in San Juanico at mooring line A ( $8 \mathrm{~m}$ depth, logger $7 \mathrm{~m}$ above bottom), line B (15 m depth, logger $13 \mathrm{~m}$ above bottom), and line $\mathrm{C}$ (3 $\mathrm{km}$ offshore line at $20 \mathrm{~m}$ depth, logger $16 \mathrm{~m}$ above the bottom) (see map in Fig. 1). (b) Bottom temperatures (logger at $1 \mathrm{~m}$ above bottom) for mooring lines A, B, and C; and zoom of water column temperatures on days 17 to 24 of August for the (c) $8 \mathrm{~m}$ mooring (site A), (d) $15 \mathrm{~m}$ mooring (site B), and (e) $20 \mathrm{~m}$ mooring (site C). Black line in zoomed water column panels denotes the $19.7^{\circ} \mathrm{C}$ isotherm, determined to be the nutricline during this period of time.

Figura 2. (a) Temperaturas subsuperficiales en San Juanico en la línea A ( $8 \mathrm{~m}$ de profundidad, sensor de temperatura a $7 \mathrm{~m}$ sobre el fondo), línea B (15 m de profundidad, sensor de temperatura a $13 \mathrm{~m}$ sobre el fondo) y línea C (3 km de la costa a $20 \mathrm{~m}$ de profundidad, sensor de temperatura a16 m sobre el fondo) (ver mapa en Fig. 1). (b) Temperaturas en el fondo (sensor de temperatura a $1 \mathrm{~m}$ sobre el fondo) para las líneas A, B y C, y ampliación de la temperatura de la columna de agua para los días del 17 al 24 de agosto para la (c) línea de 8 m (sitio A), (d) línea de $15 \mathrm{~m}$ (sitio B) y (e) línea de $20 \mathrm{~m}$ (sitio C). La línea negra en la ampliación de la columna de agua denota la isoterma de $19.7^{\circ} \mathrm{C}$, determinada a ser la nutriclina durante este periodo de tiempo. 

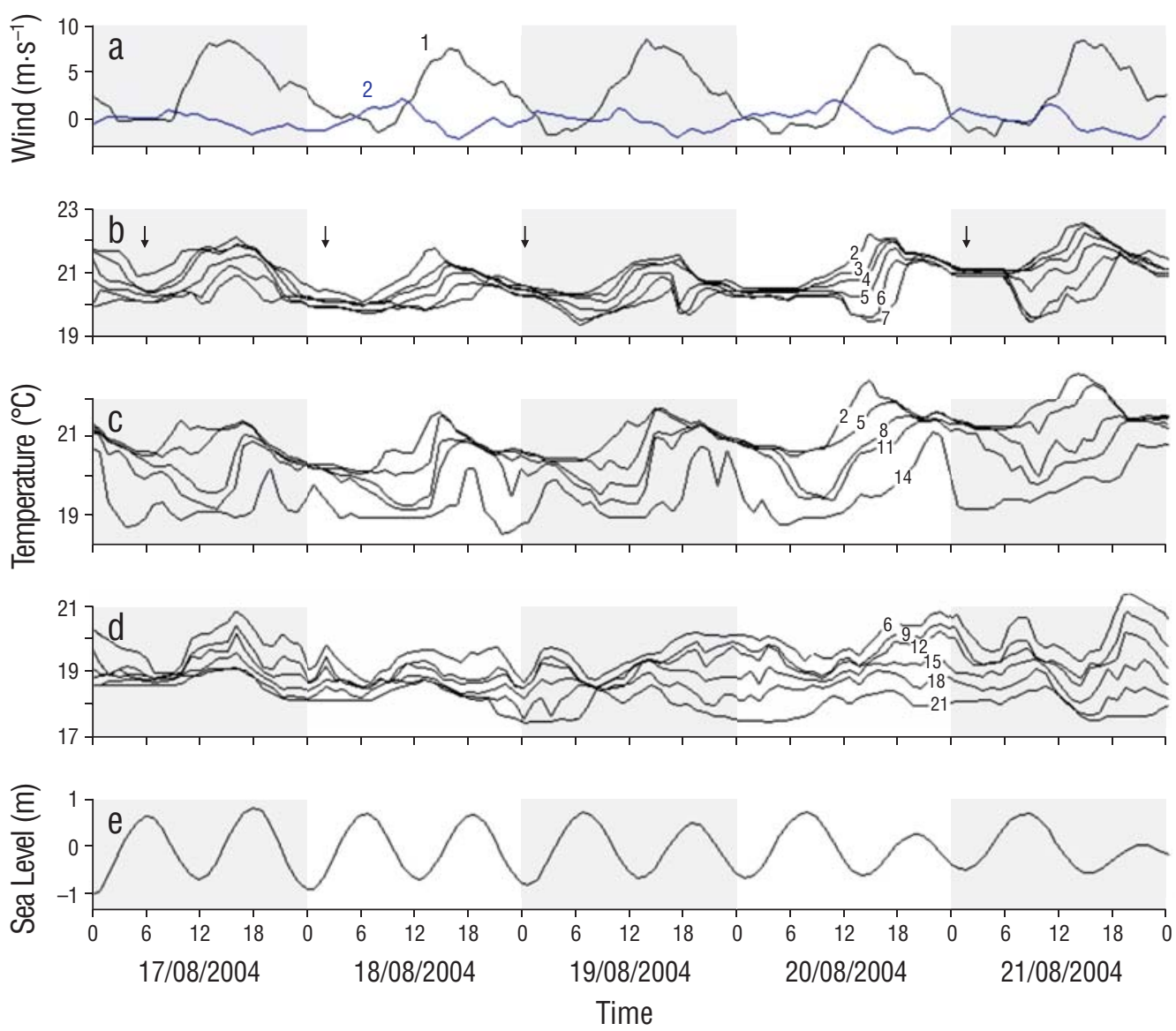

Figure 3. (a) Hourly variability of the zonal (1) and meridian (2) wind components at San Juanico. (b-d) Hourly temperature fluctuations in San Juanico at different depths on the 3 moorings (lines A, B, and C), measured on 17-21 August, and (e) predicted sea level fluctuations in San Carlos, Baja California Sur, located $160 \mathrm{~km}$ to the south of San Juanico. Vertical arrows in (b) show the times with maximum vertical mixing near line A.

Figura 3. (a) Variabilidad por hora del componente zonal (1) y meridional (2) del viento en San Juanico. (b-d) Fluctuaciones de temperatura por hora a diferentes profundidades en las 3 líneas (líneas A, B y C) en San Juanico, medidas del 17 al 21 de agosto, y (e) fluctuaciones en el nivel del mar pronosticadas para San Carlos, Baja California Sur, ubicado $160 \mathrm{~km}$ al sur de San Juanico. Las flechas verticales en (b) muestran los tiempos con mezcla vertical máxima cerca de la línea $\mathrm{A}$.

After a month of bottom temperatures with a mean of $23.6^{\circ} \mathrm{C}$ at the base of the $8-\mathrm{m}$ bed and $22.6^{\circ} \mathrm{C}$ at the base of the $15-\mathrm{m}$ bed, blades of $E$. arborea had relatively high tissue nitrogen $(2.37 \% \pm 0.19$ at $8 \mathrm{~m}, 2.40 \% \pm 0.30$ at $15 \mathrm{~m}$, maximum of $2.85 \%$ at $8 \mathrm{~m}$ ). As nutrient-limited kelp tissue has around $1 \%$ tissue nitrogen, the value measured suggests kelp tissue was not nitrogen limited at this time. Percent nitrogen, carbon $(32.39 \% \pm 1.49,31.67 \% \pm 1.68)$, or hydrogen $(3.97 \%$ $\pm 0.28,4.16 \% \pm 0.27)$ did not vary significantly between 8 and $15 \mathrm{~m}(P>0.15$, Student's $t$ test, $n=10)$.

The daytime sea breeze had maximum wind velocities $\left(8-10 \mathrm{~m} \cdot \mathrm{s}^{-1}\right)$ at $4 \mathrm{PM}$ directed to the east, with a weaker reverse night breeze (2-3 $\left.\mathrm{m} \cdot \mathrm{s}^{-1}\right)$ (Fig. 3a). At all moorings, rising temperatures occurred throughout the day as the onshore sea breeze caused accumulation of warm surface water at the coast, depressing the thermocline (Figs. 3, 4). This phase lasted from $9 \mathrm{AM}$ to $4 \mathrm{PM}$, after which time a phase of thermocline erosion occurred, with night cooling and offshore winds. The
La brisa marina durante el día presentó velocidades máximas $\left(8-10 \mathrm{~m} \cdot \mathrm{s}^{-1}\right)$ a las $4 \mathrm{PM}$ con dirección hacia el este, y hubo una inversión débil durante la noche $\left(2-3 \mathrm{~m} \cdot \mathrm{s}^{-1}\right)$ (Fig. 3a). En todas las líneas de los termistores, se registraron incrementos en la temperatura a lo largo del día cuando la brisa marina que soplaba hacia la costa provocó la acumulación de agua cálida en la superficie cerca de la costa, la cual hundió la termoclina (Figs. 3, 4). Esta fase duró desde las 9 AM hasta las 4 PM, tiempo después del cual comenzó la fase de la erosión de la termoclina, con enfriamiento nocturno y vientos que soplaban hacia afuera de la costa. La formación y erosión de la termoclina presentó la misma fase que la componente zonal del viento. La intensidad de las fluctuaciones diarias de la temperatura disminuyó de la línea A a las líneas B y C (Fig. 3b-d). En la línea C (Fig. 3d), la línea que se encontraba en mar adentro, la fase de la acumulación de calor en las capas superficiales y la iniciación del hundimiento de la termoclina 
formation and erosion of the thermocline had the same phase as the zonal wind component. The intensity of daily temperature fluctuations decreased from mooring line $\mathrm{A}$ to lines $\mathrm{B}$ and C (Fig. 3b-d). At line C (Fig. 3d), the offshore mooring, the phase accumulation of heat in the surface layers, and the timing of thermocline depression were slightly lagged compared to the coastal mooring. The hourly-averaged temperature fluctuations (Fig. 3b-d) showed a defined daily periodicity coherent with the zonal wind component (Fig. 3a) (Table 1) but were not correlated to sea level fluctuations (Fig. 3e) (Table 1).

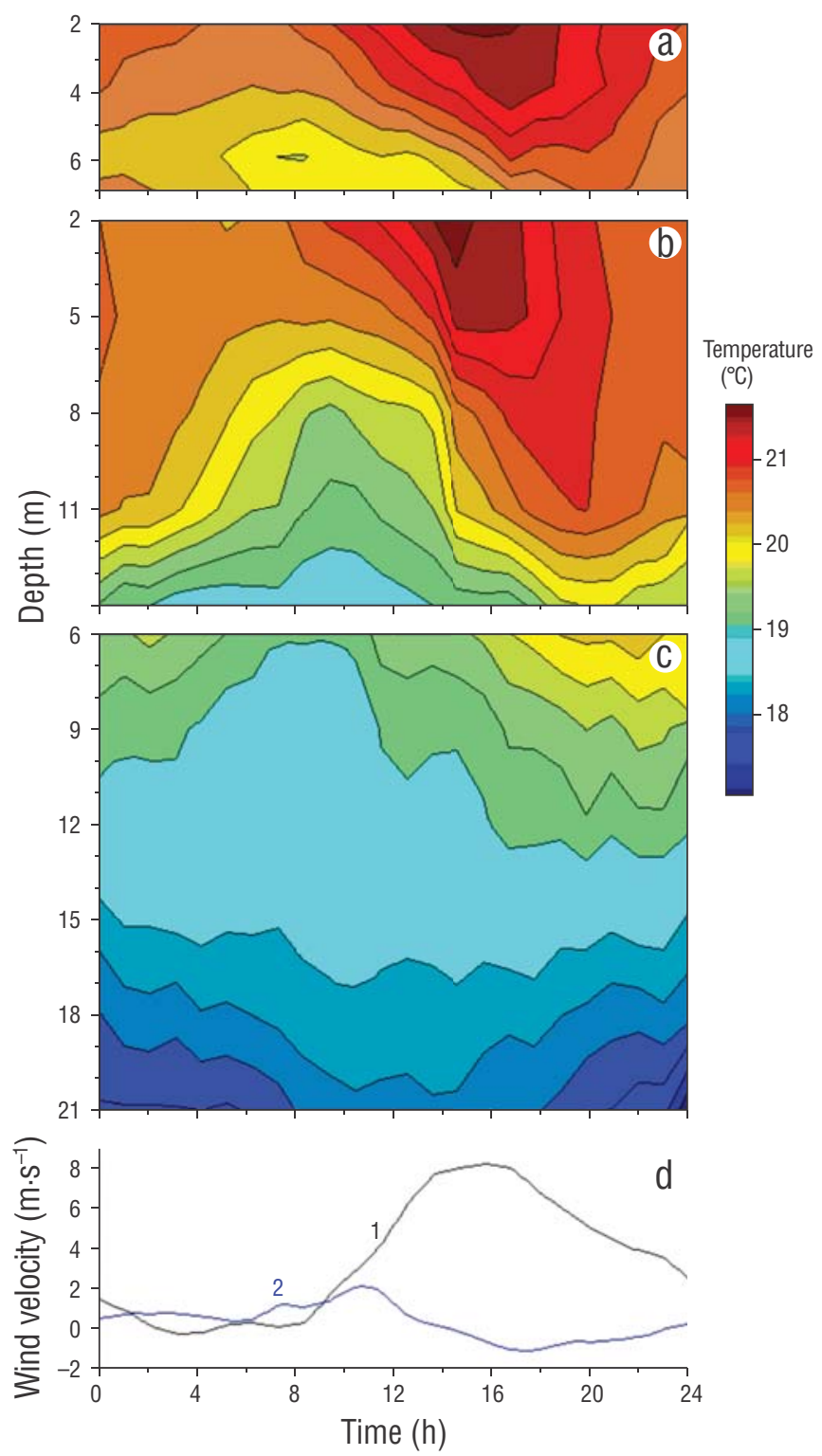

Figure 4. (a-c) Five-day average (17-21 August 2004) of temperature fluctuations at 3 moorings (a, $8 \mathrm{~m} ; \mathbf{b}, 15 \mathrm{~m} ; \mathbf{c}, 20 \mathrm{~m})$ in San Juanico bay and (d) zonal (1) and meridian (2) wind components.

Figura 4. (a-c) Promedio de 5 días (17-21 agosto de 2004) de las fluctuaciones de temperatura en las 3 líneas $(\mathbf{a}, 8 \mathrm{~m} ; \mathbf{b}, 15 \mathrm{~m}$; , $20 \mathrm{~m}$ ) en San Juanico y las componentes (d) zonal (1) y meridional (2) del viento. se presentaron más tarde con un tiempo de desfase con respecto a las líneas costeras. Las fluctuaciones de temperatura promediadas cada hora (Fig. $3 \mathrm{~b}-\mathrm{d}$ ) indicaron una periodicidad diaria definida coherente con la componente zonal del viento (Fig. 3a) (Tabla 1) pero no estuvieron correlacionadas con las fluctuaciones en el nivel del mar (Fig. 3e) (Tabla 1).

Los mapas comareales de las 4 ondas fundamentales, $\mathrm{O}_{1}$ (25.8 h), $\mathrm{K}_{1}(23.9 \mathrm{~h}), \mathrm{M}_{2}(12.4 \mathrm{~h}) \mathrm{y} \mathrm{S}_{2}(12.0 \mathrm{~h})$, para la costa oeste de la península de Baja California (Godin et al. 1980) indican que la amplitud de la marea semidiurna es casi el doble de la marea diurna para esta región (Fig. 5). Lo opuesto se encontró para el espectro de las fluctuaciones de temperatura medidas en las líneas, donde el pico diurno era de un orden de magnitud mayor que el pico semidiurno, lo que sugiere que las fluctuaciones diarias en la temperatura del agua cerca de la costa no fueron provocadas por la marea (Fig. 5).

El cuadrado de la coherencia de la variabilidad de la temperatura en todas las profundidades de la línea A y el componente dominante este-oeste del viento era casi 2 veces más grande que el intervalo de confianza (IC) en el periodo diurno (Tabla 1). Para la línea más profunda (línea C), solo las capas superficiales (los primeros $7 \mathrm{~m}$ de la columna de agua) presentaron coherencia significativa con la energía diurna del viento arriba del IC del $95 \%$ y disminuyeron a casi cero (0.034) en el fondo. Alrededor de la línea A (300 m de la costa, en aproximadamente $7 \mathrm{~m}$ de profundidad), el tiempo de desfase entre la velocidad máxima del viento y el hundimiento de la termoclina hasta el fondo fue de aproximadamente $4 \mathrm{~h}$. Alrededor de la línea B $(2.5 \mathrm{~km}$ de la costa, en aproximadamente $14 \mathrm{~m}$ de profundidad), el tiempo de desfase era de casi $6 \mathrm{~h}$. No hubo coherencia significativa entre la temperatura y la marea semidiurna (12.42 h) (Fig. 6,

Table 1. Squared coherence values between the dominant E-W (U) component of the wind and temperature fluctuations, and between the sea level and temperature fluctuations. Calculations are shown for thermistors on mooring line A, coastal kelp bed, $8 \mathrm{~m}$ depth. Values above the $95 \%$ confidence interval $(0.521)$ are shown in bold.

Tabla 1. Valor de la coherencia cuadrada entre el componente dominante $\mathrm{E}-\mathrm{W}(\mathrm{U})$ del viento y las fluctuaciones de temperatura, y entre el nivel del mar y las fluctuaciones de temperatura. Se muestran los cálculos para la línea de termistores A, manto de kelp costero, a $8 \mathrm{~m}$ de profundidad. Los valores arriba del intervalo de confianza del $95 \%$ (0.521) se muestran en negritas.

\begin{tabular}{lccccccc}
\hline & & \multicolumn{6}{c}{ Thermistors (height above the bottom, m) } \\
\cline { 3 - 8 } Period (h) & variable & 2 & 3 & 4 & 5 & 6 & 7 \\
\hline $\begin{array}{l}12.42 \\
\mathrm{M}_{2}\end{array}$ & Sea level & 0.200 & 0.238 & 0.343 & 0.387 & 0.324 & 0.337 \\
25.82 & & & & & & & \\
$\mathrm{O}_{1}$ & Sea level & 0.167 & 0.289 & 0.439 & 0.334 & 0.177 & 0.278 \\
24.00 & Wind & $\mathbf{0 . 7 7 2}$ & $\mathbf{0 . 8 3 3}$ & $\mathbf{0 . 8 3 5}$ & $\mathbf{0 . 8 6 5}$ & $\mathbf{0 . 8 1 4}$ & $\mathbf{0 . 7 7 4}$ \\
\hline
\end{tabular}


Cotidal maps for the 4 basic tidal waves, $\mathrm{O}_{1}(25.8 \mathrm{~h}), \mathrm{K}_{1}$ (23.9 h), $\mathrm{M}_{2}(12.4 \mathrm{~h})$, and $\mathrm{S}_{2}(12.0 \mathrm{~h})$, for the western coast of the Baja California Peninsula (Godin et al. 1980) show that the amplitude of the semidiurnal tide is almost twice that of the diurnal tide for this area (Fig. 5). The reverse was found for the spectrum of mooring temperature fluctuations, however, where the diurnal peak was one order of magnitude greater than the semidiurnal peak, suggesting that daily water temperature fluctuations near the coast were not caused by the tide (Fig. 5).

The squared coherence between the temperature variability at all depths on mooring line A and the dominant EastWest component of the wind was almost twice as large as the 95\% confidence level (CI) in the diurnal period (Table 1). For the deeper offshore mooring (line C), only the surface layers (top $7 \mathrm{~m}$ of the water column) were significantly coherent with diurnal wind energy above the $95 \% \mathrm{CI}$ and dropped to near zero (0.034) at depth. In the area of mooring A (300 $\mathrm{m}$ from the shore, in approximately $7 \mathrm{~m}$ depth), the
Tabla 1). Esto sugiere que los vientos diurnos locales provocan las fluctuaciones de temperatura observadas en los mantos de kelp, en contraste con otros sitios en Baja California donde el forzamiento por la marea semidiurna ha mostrado ser más importante (Ladah et al. 2012, Filonov et al. 2014).

\section{Discusión}

Los mantos de kelp son comunes en la corriente de California, pero alcanzan su límite sur en la parte sureña de este ecosistema, en las áreas frente a Baja California Sur, donde las surgencias se vuelven intermitentes. La concentración relativamente elevada de nitrógeno en el kelp, E. arborea (hasta 2.85\%), medida después de exposición a agua cálida durante un mes en verano (temperatura promedio del fondo de $23^{\circ} \mathrm{C}$ ) en San Juanico, junto con datos previos que muestran la respuesta de E. arborea ante bajas concentraciones de nitrógeno durante $20 \mathrm{~d}$ tanto en cultivos de tanque como en el campo (Matson y Edwards 2007, Sánchez-Barredo et al.

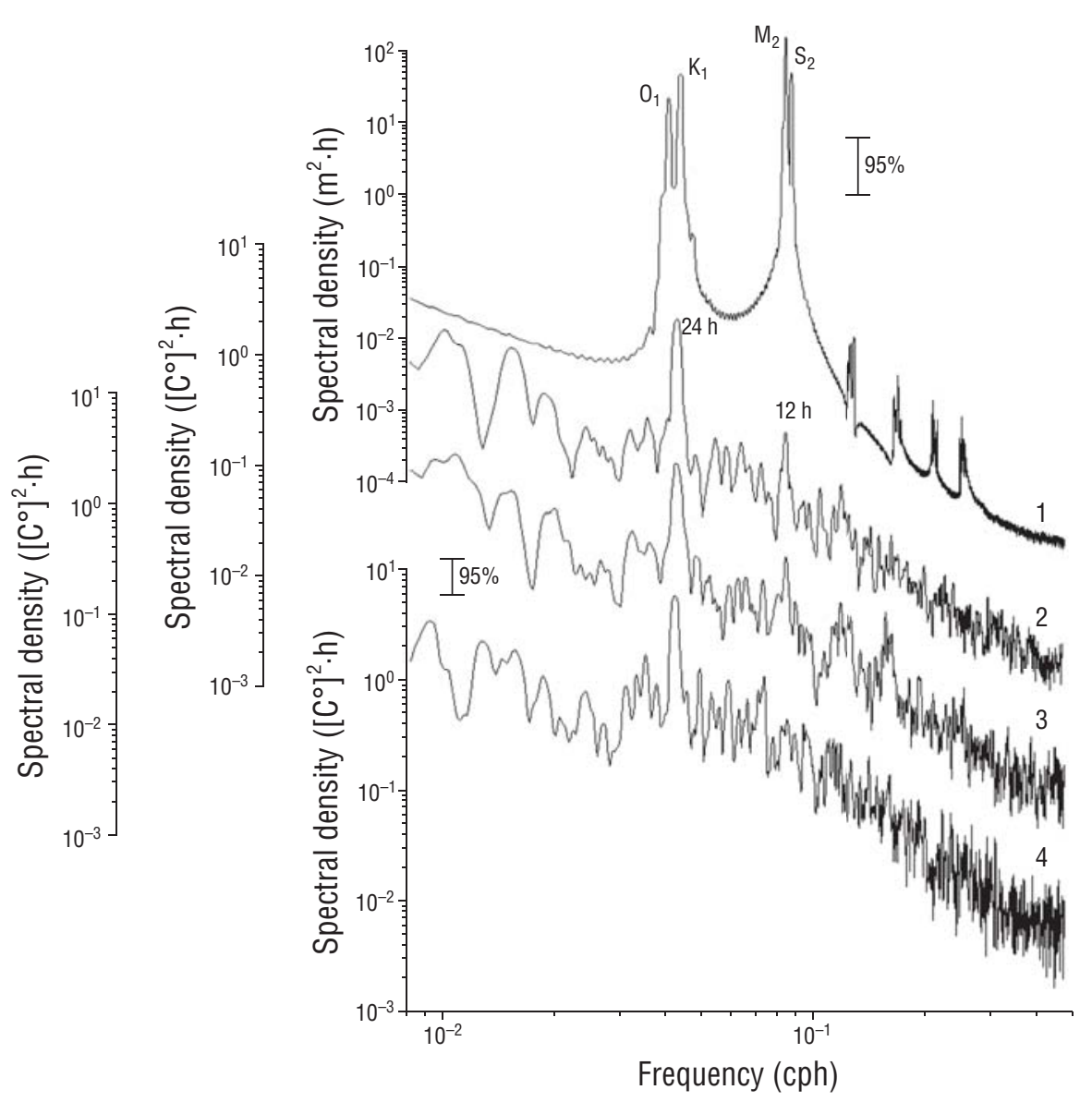

Figure 5. Spectral density of sea level fluctuations in San Carlos (1) and average spectral density of temperature fluctuations at mooring lines A (2), B (3), and C (4), calculated for temperature data collected at all measurement depths. Vertical error bar indicates the $95 \%$ confidence interval for the power spectral density function.

Figura 5. Densidad espectral de las fluctuaciones del nivel del mar para San Carlos (1) y densidad espectral promedio de las fluctuaciones de temperatura en las líneas A (2), B (3) y C (4), calculada para todos los datos de temperatura medidos a todas las profundidades. La barra de error vertical indica el intervalo de confianza del $95 \%$ para la función de densidad espectral de potencia. 
phase lag between the maximum wind speed and the depression of the thermocline down to the bottom was about $4 \mathrm{~h}$. In the area of mooring B (2.5 km from the shore, at a depth of approximately $14 \mathrm{~m}$ ), this lag was almost $6 \mathrm{~h}$. There was no significant coherence between temperature and the semidiurnal (12.42 h) tide (Fig. 6, Table 1). This suggests that local diurnal winds cause the observed temperature fluctuations in the kelp beds, in contrast to others sites off Baja California, where semidiurnal tidal forcing has been shown to be more important (Ladah et al. 2012, Filonov et al. 2014).

\section{Discussion}

Kelp beds are common in the California Current, but reach their southern limit in the southern part of this ecosystem in areas off Baja California Sur, where upwelling becomes intermittent. The relatively high tissue nitrogen concentration in the kelp, E. arborea (up to $2.85 \%$ ), measured after a month of warm water in summer (bottom temperature mean of $23{ }^{\circ} \mathrm{C}$ ) in San Juanico, along with previous data showing the kelp species $E$. arborea responds to low nitrogen conditions within $20 \mathrm{~d}$ both in tank cultures and in the field (Matson and Edwards 2007, Sánchez-Barredo et al. 2011), suggests a recent source of external inorganic nitrogen reached the kelp beds. The measurement of nitrate and nitrite in water as warm as $19.7^{\circ} \mathrm{C}$, the short diurnal intrusions of water cooler than $19^{\circ} \mathrm{C}$ into the kelp beds even when sea surface temperatures were high, and the coherence of intrusions with daily winds suggest that diurnal internal waves forced by winds may transport nutrients to these kelp beds during warm periods.

Coastal nitrate enrichment at $18.5^{\circ} \mathrm{C}$ has been previously shown for the California Current off Baja California in Tortugas Bay (Hernández-Carmona et al. 2001), and an analysis of older California Cooperative Oceanic Fisheries Investigations data has suggested that anomalous warm-water nitrate enrichment might occur during El Niño events (Ladah 2003). However, in the present study, nitrate was detected at even higher temperatures (at almost $20^{\circ} \mathrm{C}$ ) at a site further south in the California Current than previously analyzed. In a previous study, Ladah (2003) showed a latitudinal increase in nitrate enrichment (with $19.5^{\circ} \mathrm{C}$ water having $1.1 \mu \mathrm{M}$ nitrate, at a site $100 \mathrm{~km}$ to the north of San Juanico) in warm waters during El Niño and speculated this was due to the shoaling of subtropical subsurface water. The present study also occurred during an El Niño event (20042005), further supporting the idea that nutrient-rich water can shoal during El Niño in Baja California Sur. However, the persistence, seasonality, and source of enrichment have still not been identified.

Diurnal temperature changes were detected within the kelp beds during this study and showed significant coherence with the diurnal frequency in winds. Although diurnal internal waves are not unprecedented in the California Current and have been previously documented in California (Lerczak et al. 2001, Pidgeon and Winant 2005, Beckenbach and Terrill
2011), sugiere que una fuente de nitrógeno inorgánico externa y reciente alcanzó los mantos de kelp. La medición de nitrato y nitrito en agua cálida con temperaturas de hasta $19.7^{\circ} \mathrm{C}$, las intrusiones cortas y diurnas de agua más fría que $19^{\circ} \mathrm{C}$ en los mantos de kelp aun cuando las temperaturas en la superficie eran elevadas y la coherencia entre las intrusiones y los vientos diarios sugieren que las ondas internas diurnas forzadas
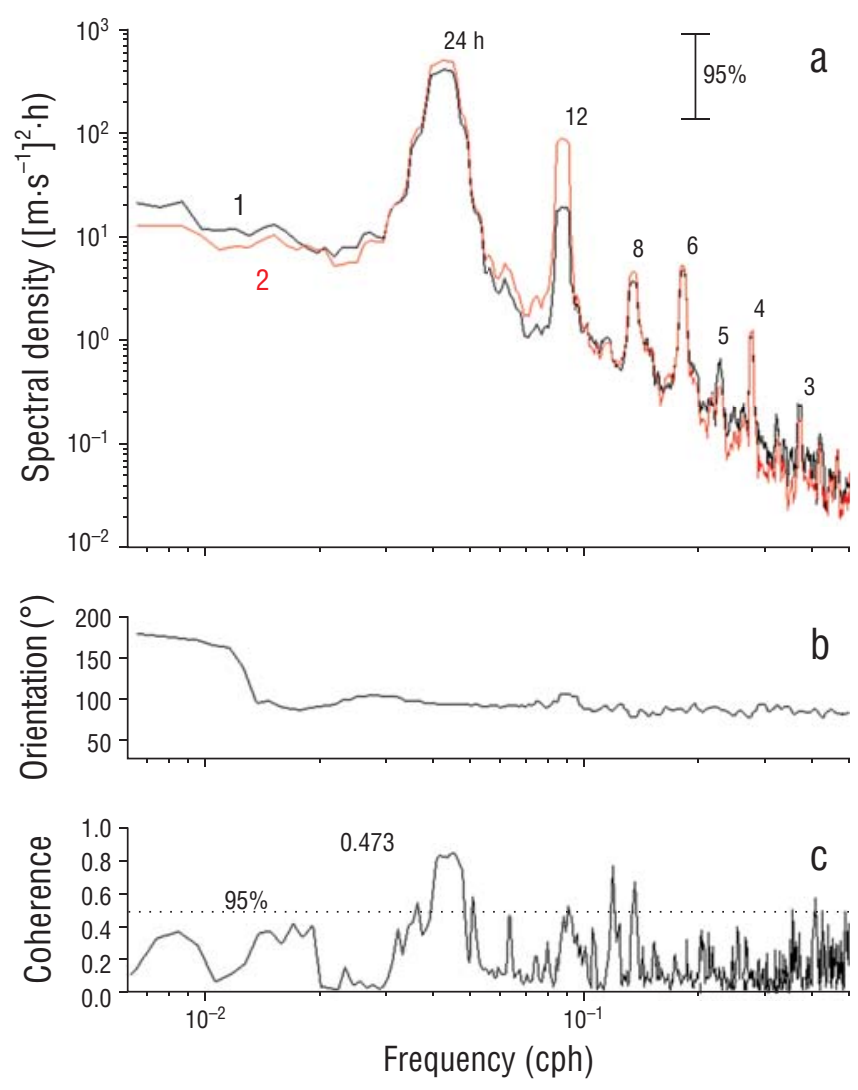

Figure 6. (a) Clockwise (1) and counterclockwise (2) rotational spectra of wind variability at San Juanico. Vertical error bar indicates the $95 \%$ confidence interval for the power spectral density function. (b) Main axis orientation for the elliptical orbits, calculated in degrees from North clockwise. (c) Coherence between temperature variability at the surface at mooring line A and variability of the wind energy (sum of the zonal and meridional wind components). Dotted vertical line indicates the $95 \%$ confidence interval for the squared coherence.

Figura 6. (a) Espectro rotacional de la variabilidad del viento en San Juanico en sentido de las agujas del reloj (1) y en contra del sentido de las agujas del reloj (2). La barra de error vertical indica el intervalo de confianza del $95 \%$ para la función de la densidad espectral de potencia. (b) Eje principal de orientación para las orbitas elípticas, calculado en grados del norte en sentido de las agujas del reloj. (c) Coherencia entre la variabilidad de la temperatura en la superficie en la línea A y la variabilidad de la energía del viento (suma de los componente zonal y meridional del viento). La línea punteada indica el intervalo de confianza del $95 \%$ para el cuadrado de la coherencia. 
2008, Cudaback and McPhee-Shaw 2009), we expected to find stronger semidiurnal variability in our temperature time series similar to what has been found for other sites in Mexico. The southern subtropical part of the California Current encompasses latitudes where theory predicts that unforced, freely propagating, diurnal internal waves can exist, as they are not subinertial at these latitudes. However, off the west coast of Mexico, the barotropic tide is a mixed semidiurnal tide, often resulting in a much larger variance at the semidiurnal frequency in nearshore water column temperatures than at the diurnal frequency (Filonov and Trasviña 2000; Filonov and Konyaev 2003, 2006; Filonov and Novotryasov 2005; Ladah et al. 2005; Filonov et al. 2014). Because San Juanico is located near the edge of a long shallow shelf in the Gulf of Ulloa (200 m isobath, see Fig. 1), where internal tides could be generated (Baines 1982, Holloway 1991, Konyaev and Sabinin 1992) approximately $120-150 \mathrm{~km}$ from the coastline, we expected tidally forced, semidiurnal internal waves to reach the coast, with wave lengths in the range of hundreds of meters and frequencies close to the maximum buoyancy frequency (periods of 3-15 min). We did not expect winds to have a greater forcing effect than the semidiurnal tide, certainly not at the base of the kelp beds, as most areas studied to date in the Mexican Pacific show a strong semidiurnal internal tide, except very far inside Todos Santos Bay (Filonov et al. 2014), where weak diurnal internal motions occur trapped to the coast.

We propose that in San Juanico, semidiurnal internal waves are generated offshore, but dissipate on the long shallow shelf before reaching the kelp beds inshore, where wind-forced diurnal internal waves become more prevalent. Because mesoscale upwelling of nutrient-rich water can be weak and intermittent in summer off Baja California Sur (Kurzyn et al. 2012), the transport of nutrients into shallow zones by the daily thermocline shoaling measured in this study may be particularly relevant for southern-limit kelp beds. Although the percentage of overall nutrient flow to the system that diurnal internal waves can provide to this site is currently unknown, this topic merits further study as a mechanism to sustain primary productivity, particularly during upwelling-limited summers and El Niño years.

\section{ACKNOWLedgments}

Special thanks to Dr. Gilo Gaxiola ${ }^{\dagger}$ for the many enriching discussions on internal waves and their importance to the productivity of the California Current. The authors acknowledge UC MEXUS, IAI-EPCOR, SEP-CONACYT (50046), AMELIS-CONACYT (622167), the Puerto Chale and San Juanico Fishing Cooperatives, and the Scorpion Bay Campground. Andrés Ortinez, Juan de la Cruz, Shinquiro (Niko), Giovanni, Daviel, Kaliman, and Pedro Aguilar helped with the field work. A very special thank you to Ruben, Dave, and Laurie, and to the Interdisciplinary Coastal Ecology team at CICESE. por el viento podrían transportar nutrientes a los mantos de kelp durante periodos cálidos.

El enriquecimiento de nitrato cerca de la costa a $\operatorname{los} 18.5^{\circ} \mathrm{C}$ ha sido reportado previamente para la corriente de California en bahía Tortugas en Baja California (Hernández-Carmona et al. 2001), y un análisis de datos del programa California Cooperative Oceanic Fisheries Investigations sugiere que el enriquecimiento anómalo de nitrato en agua cálida podría ocurrir durante eventos de El Niño (Ladah 2003). Sin embargo, en este estudio, se detectaron concentraciones de nitrato a temperaturas aún más elevadas (casi $20^{\circ} \mathrm{C}$ ) en un sitio aún más al sur en la corriente de California de lo que se ha analizado previamente. En un estudio previo, Ladah (2003) mostró un incremento latitudinal en el enriquecimiento de nitrato (agua de $19.5^{\circ} \mathrm{C}$ con una concentración de nitrato de $1.1 \mu \mathrm{M}$, en un sitio $100 \mathrm{~km}$ al norte de San Juanico) en aguas cálidas durante un evento El Niño y especuló que esto se debió a que el agua subsuperficial subtropical estaba a profundidades más someras que en años normales. El presente estudio también se llevó a cabo durante un evento El Niño (2004-2005), lo que apoya aún más la idea de que el bombeo de aguas profundas ricas en nutriente hacia aguas más someras puede suceder durante condiciones de El Niño en Baja California Sur. Sin embargo, la persistencia, la temporalidad y la fuente de enriquecimiento aún no han sido identificadas.

Los cambios diurnos de temperatura fueron detectados dentro de los mantos de kelp durante este estudio y mostraron coherencia significativa con la frecuencia diurna de los vientos. A pesar de que las ondas internas diurnas han sido documentadas previamente en la corriente de California (Lerczak et al. 2001, Pidgeon y Winant 2005, Beckenbach y Terrill 2008, Cudaback y McPhee-Shaw 2009), esperábamos encontrar que la variabilidad semidiurna fuese más fuerte en nuestra serie de tiempo de temperatura, parecido a lo que se ha encontrado en otros sitios en México. La parte sur subtropical de la corriente de California abarca latitudes donde la teoría predice que ondas internas diurnas que propagan libremente pueden existir, dado que no son subinerciales a estas latitudes. Sin embargo, frente a la costa occidental de México, la marea barotrópica es una marea semidiurna mixta, que frecuentemente resulta en una varianza mayor en la temperatura de la columna de agua cerca de la costa en la frecuencia semidiurna que en la frecuencia diurna (Filonov y Trasviña 2000; Filonov y Konyaev 2003, 2006; Filonov y Novotryasov 2005; Ladah et al. 2005; Filonov et al. 2014). Dado que San Juanico se encuentra localizado cerca de la orilla de la plataforma larga y somera del golfo de Ulloa (isóbata de $200 \mathrm{~m}$, ver Fig. 1), donde podrían generarse mareas internas (Baines 1982, Holloway 1991, Konyaev y Sabinin 1992) a aproximadamente 120-150 km de la línea de costa, esperábamos observar que las ondas internas semidiurnas forzadas por la marea llegaran hasta la costa, con longitudes de onda de cientos de metros y con frecuencias cercanas a la frecuencia máxima de flotabilidad (periodos de 3-15 min). No esperábamos observar que los vientos ejercieran un efecto de forzamiento 


\section{REFERENCES}

Apel JR, Gonzalez FI. 1983. Nonlinear features of internal waves off Baja California as observed from the SEASAT imaging radar. J. Geophys. Res. 88(C7): 4459-4466. https://doi.org/10.1029/jc088ic07p04459

Baines PG. 1982. On internal tide generation models. Deep-Sea Res. 29(3): 307-338. https://doi.org/10.1016/0198-0149(82)90098-X

Beckenbach E, Terrill E. 2008. Internal tides over abrupt topography in the Southern California Bight: Observations of diurnal waves poleward of the critical latitude. J. Geophys. Res. 113(C2): C02001. https://doi.org/10.1029/2006jc003905

Cudaback CN, McPhee-Shaw E. 2009. Diurnal-period internal waves near point conception, California. Estuar. Coast. Shelf Sci. 83(3): 349-359. https://doi.org/10.1016/j.ecss.2008.12.018

Emery WJ, Thomson RE. 1997. Data Analysis Methods in Physical Oceanography. Elsevier, Amsterdam. 638 pp.

Filonov AE, Konyaev KV. 2003. Nonlinear internal waves near the Mexico's Central Pacific Coast. In: Velasco-Fuentes OU, Scheinbaum J, Ochoa J (eds.), Nonlinear Processes in Geophysical Fluid Dynamics. Kluwer Academic Publishers, Netherlands, pp. 377-386.

Filonov A, Konyaev K. 2006. Orbital motions and coherent structures in the internal tide on a steep shelf off the pacific coast of Mexico. Geophys. Res. Lett. 33(14): L14612. https://doi.org/10.1029/2006gl026712

Filonov A, Lavín MF, Ladah LB, Tereshchenko I. 2014. Spatial variability of internal waves in an open bay with a narrow steep shelf in the Pacific off NW Mexico. Cont. Shelf Res. 78: 1-15. https://doi.org/10.1016/j.csr.2014.01.015

Filonov A, Novotryasov V. 2005. Features of the nonlinear wave spectrum in the coastal zone. Geophys. Res. Lett. 32(15): L15602. https://doi.org/10.1029/2005gl023046

Filonov AE, Trasviña A. 2000. Internal waves on the continental shelf of the Gulf of Tehuantepec, Mexico. Estuar. Coast. Shelf Sci. 50(4): 531-548. https://doi.org/10.1006/ecss.1999.0583

Franks PJS. 1992. Sink or swim: accumulation of biomass at fronts. Mar. Ecol. Progr. Ser. 82: 1-12. https://doi.org/10.3354/meps082001

Franks PJS. 1997. New models for the exploration of biological processes at fronts. ICES J. Mar. Sci. 54(2): 161-167. https://doi.org/10.1006/jmsc.1996.0191

Gerard VA. 1982. Growth and utilization of internal nitrogen reserves by the giant kelp Macrocystis pyrifera in a low nitrogen environment. Marine Biology 66(1): 27-35. https://doi.org/10.1007/bf00397251

Gerard VA. 1984. Physiological effects of El Niño on giant kelp in Southern California. Mar. Biol. Lett. 5: 317-322.

Gerard VA. 1997. The role of nitrogen nutrition in high-temperature tolerance of the kelp Laminaria saccharina (Chromophyta). J. Phycol. 33(5): 800-810. https://doi.org/10.1111/j.0022-3646.1997.00800.x

Godin G, De la Paz R, Rodríguez N, Ortiz M. 1980. La marea y el nivel del mar a lo largo de la costa occidental de México. Geofís. Int. 19(3): 239-258.

Gonella J. 1972. A rotary-components method for analysing meteorological and oceanographic vector time series. Deep-Sea Res. 19(12): 833-846. https://doi.org/10.1016/0011-7471(72)90002-2

Helfrich KR, Pineda J. 2003. Accumulation of particles in propagating fronts. Limnol. Oceanogr. 48(4): 1509-1520. https://doi.org/10.4319/1o.2003.48.4.1509 mayor que la marea semidiurna, especialmente en la base de los mantos de kelp, ya que la mayoría de las áreas estudiadas en México presentan una fuerte marea interna semidiurna, a excepción de la parte interna de la bahía de Todos Santos (Filonov et al. 2014), donde las ondas internas diurnas son débiles y están atrapadas en la costa.

Proponemos que en San Juanico, las ondas internas semidiurnas se generan mar adentro pero se disipan a lo largo de la plataforma larga y somera antes de alcanzar los mantos de kelp cerca de la costa, donde las ondas internas diurnas forzadas por el viento se vuelvan más prevalentes. Dado que las surgencias a mesoescala de aguas ricas en nutrientes pueden ser débiles e intermitentes durante el verano en Baja California Sur (Kurzyn et al. 2012), el transporte de nutrientes hacia aguas someras por ondas internas diurnas podría ser particularmente importante para los mantos de kelp en los límites sureños de su distribución. A pesar de que el porcentaje total de nutrientes suministrado al sistema por las ondas internas diurnas en este sitio actualmente se desconoce, este tema merita más investigación para evaluar la capacidad de este mecanismo para sostener la productividad primaria, particularmente durante los veranos cuando la actividad de surgencias es limitada y durante los eventos de El Niño.

\section{Agradecimientos}

Un agradecimiento especial al Dr. Gilo Gaxiola ${ }^{\dagger}$ por todas las conversaciones enriquecedoras sobre las ondas internas y su importancia para la productividad de la corriente de California. Los autores reconocen a UC MEXUS, IAI-EPCOR, SEP-CONACYT (50046), AMELIS-CONACYT (622167), las cooperativas pesqueras de San Juanico y puerto Chale, y el campamento Scorpion Bay. Andrés Ortinez, Juan de la Cruz, Shinquiro (Niko), Giovanni, Daviel, Kaliman y Pedro Aguilar ayudaron con el trabajo de campo. Un agradecimiento especial a Ruben, Dave y Laurie, y al equipo del laboratorio Interdisciplinary Coastal Ecology del CICESE.

Helfrich KR, Melville WK. 2006. Long nonlinear internal waves. Annu. Rev. Fluid Mech. 38: 395-425.

Hernández-Carmona G, Robledo D, Serviere-Zaragoza E. 2001. Effect of nutrient availability on Macrocystis pyrifera recruitment and survival near its southern limit off Baja California. Bot. Mar. 44(3): 221-229.

https://doi.org/10.1515/bot.2001.029

Holloway PE. 1987. Internal hydraulic jumps and solitons at a shelf break region on the Australian Northwest shelf. J. Geophys. Res. Oceans 92(C5): 5404-5416. https://doi.org/10.1029/jc092ic05p05405

Holloway P. 1991. On the dissipation of internal tides. In: Parker BB (ed.), Tidal Hydrodynamics. Wiley, Rockvile, Maryland, USA.

Konyaev KV, Sabinin KD. 1992. Waves Inside the Ocean. Hydrometeoizdat, St. Petersburg, $272 \mathrm{pp}$.

Konyaev KV. 1990. Spectral Analysis of Physical Oceanographic Data. A.A. Balkema, Rotterdam, Amsterdam, 200 pp.

Kurczyn JA, Beier E, Lavín MF, Chaigneau A. 2012. Mesoscale eddies in the northeastern Pacific tropical-subtropical transition zone: Statistical characterization from satellite 
altimetry. J. Geophys. Res. 117(C10): C10021.

https://doi.org/10.1029/2012jc007970

Ladah LB. 2003. The shoaling of nutrient-enriched subsurface water as a mechanism to sustain primary productivity off Central Baja California during El Niño winters. J. Mar. Systems 42(3-4): 145-152. https://doi.org/10.1016/s0924-7963(03)00072-1

Ladah LB, Filonov A, Lavín M, Leichter J, Zertuche-González J, Pérez-Mayorga D. 2012. Cross shelf transport of sub-thermocline nitrate by the internal tide and rapid (3-6 h) incorporation by an inshore macroalga. Cont. Shelf Res. 42: 10-19.

https://doi.org/10.1016/j.csr.2012.03.010

Ladah LB, Tapia FJ, Pineda J, Lopez M. 2005. Spatially heterogeneous synchronous settlement of Chthamalus spp. larvae in Northern Baja California. Mar. Ecol. Prog.Ser. 302: 177-185. https://doi.org/10.3354/meps302177

Lamb KG. 1997. Particle transport by nonbreaking solitary internal waves. J. Geophys. Res. 102(C8): 18641-18660. https://doi.org/10.1029/97jc00441

Leichter JJ, Stewart HL, Miller SL. 2003. Episodic nutrient transport to Florida coral reefs. Limnol. Oceanogr. 48(4): 1394-1407. https://doi.org/10.4319/lo.2003.48.4.1394

Leichter JJ, Wing SR, Miller SL, Denny MW. 1996. Pulsed delivery of subthermocline water to Conch Reef (Florida Keys) by internal tidal bores. Limnol. Oceanogr. 41(7): 1490-1501. https://doi.org/10.4319/1o.1996.41.7.1490

Lerczak JA, Hendershott MC, Winant CD. 2001. Observations and modeling of coastal internal waves driven by a diurnal sea breeze. J. Geophys. Res. 106(C9): 19715-19729. https://doi.org/10.1029/2001jc000811

Lucas AJ, Dupont CL, Tai V, Largier JL, Palenik B, Franks PJS. 2011. The green ribbon: multiscale physical control of phytoplankton productivity and community structure over a narrow continental shelf. Limnol. Oceanogr. 56(2): 611-626. https://doi.org/10.4319/lo.2011.56.2.0611

Matson PG, Edwards MS. 2007. Effects of ocean temperature on the southern range limits of two understory kelps, Pterygophora californica and Eisenia arborea at multiple life-stages. Marine Biology 151(5): 1941-1949. https://doi.org/10.1007/s00227-007-0630-

McPhee-Shaw EE, Siegel DA, Washburn L, Brzezinski MA, Jones JL, Leydecker AI, Melack J. 2007. Mechanisms for nutrient delivery to the inner shelf: observations from the Santa Barbara Channel. Limnol. Oceanogr. 52(5): 17481756. https://doi.org/10.4319/1o.2007.52.5.1748

Pérez-Mayorga D, Ladah LB, Zertuche-González J, Leichter JJ, Filonov AE, Lavín MF. 2011. Nitrogen uptake and growth by the opportunistic macroalga Ulva lactuca (Linnaeus) during the internal tide. J. Exp. Mar. Biol. Ecol. 406(12): 108-115. https://doi.org/10.1016/j.jembe.2011.05.028
Pidgeon EJ, Winant CD. 2005. Diurnal variability in currents and temperature on the continental shelf between central and southern California. J. Geophys. Res. 110(C3): C03024. https://doi.org/10.1029/2004jc002321

Salas-Monreal D, Salas-de-Leon DA, Monreal-Gomez MA, RiverónEnzástiga ML, Mojica-Ramírez E. (2012). Hydraulic jump in the Gulf of California. Open J. Mar. Sci. 2(4): 141-149. https://doi.org/10.4236/ojms.2012.24017

Sánchez-Barredo M, Ladah LB, Zertuche-González JA. 2011. Nitrate uptake and duration of internal nitrogen reserves in the kelp Eisenia arborea. Bot. Mar. 54(5): 441-446. https://doi.org/10.1515/bot.2011.058

Sandstrom H, Elliott JA. 1984. Internal tide and solitons on the Scotian shelf: a nutrient pump at work. J. of Geophys. Res. 89(C4): 6415-6426. https://doi.org/10.1029/jc089ic04p06415

Sandstrom, H, Elliott JA. 2011. Production, transformation, and dissipation of energy in internal tides near the continental shelf edge. J. Geophys. Res. 116(C4): C04004. http://dx.doi.org/10.1029/2010JC006296

Schafstall J, Dengler M, Brandt P, Bange H. 2010. Tidal-induced mixing and diapycnal nutrient fluxes in the Mauritanian upwelling region. J Geophys. Res. 115(C10): C10014. https://doi.org/10.1029/2009jc005940

Sharples J, Moore CM, Abraham ER. 2001. Internal tide dissipation mixing and vertical nitrate flux at the shelf edge of NE New Zealand. J. Geophys. Res. 106(C7): 14069-14081. https://doi.org/10.1029/2000jc000604

Sharples J, Moore CM, Hickman AE, Holligan PM, Tweddle JF, Palmer MR, Simpson JH. 2009. Internal tidal mixing as a control on continental margin ecosystems. Geophys. Res. Lett. 36(23): L23603. https://doi.org/10.1029/2009gl040683

Sharples J, Tweddle JF, Green JA, Palmer MR, Kim YN, Hickman AE, Holligan PM, Moore CM, Rippeth TP, Simpson JH, Krivtsov V. 2007. Spring neap modulation of internal tide mixing and vertical nitrate fluxes at a shelf edge in summer. Limnol. Oceanogr. 52(5): 1735-1747. https://doi.org/10.4319/1o.2007.52.5.1735

Zentara SJ, Kamykowski D. 1977. Latitudinal relationships among temperature and selected plant nutrients along the west coast of North and South America. J. Mar. Res. 35: 321-337.

Zimmerman RC, Kremer NJ. 1984. Episodic nutrient supply to a kelp forest ecosystem in Southern California. J. Mar. Res. 42(3): 591-604. https://doi.org/10.1357/002224084788506031

Zimmerman RC, Kremer NJ. 1986. In situ growth and chemical composition of the giant kelp, Macrocystis pyrifera: response to temporal changes in ambient nutrient availability. Mar. Ecol. Prog. Ser. 27: 277-285. https://doi.org/10.3354/meps027277

Received April 2017, Accepted October 2017. 